Artículos

ESTUDIOS SOCIALES

\title{
Escalas y gobierno en el estudio de la nueva derecha argentina
}

\author{
Scales and government in the study of the new argentine \\ right
}

Salerno, Agustín

Agustín Salerno agustinnsalerno@gmail.com Facultad Latinoamericana de Ciencias Sociales Consejo de Investigaciones Científicas y Técnicas (FLACSO - CONICET), Argentina

\author{
Estudios Sociales. Revista Universitaria Semestral \\ Universidad Nacional del Litoral, Argentina \\ ISSN: 0327-4934 \\ ISSN-e: 2250-6950 \\ Periodicidad: Semestral \\ núm. 61, e0009, 2021 \\ estudiossociales@unl.edu.ar
}

Recepción: 29 Octubre 2020

Aprobación: 25 Junio 2021

URL: http://portal.amelica.org/ameli/jatsRepo/293/2932743011/ index.html

DOI: https://doi.org/10.14409/es.2021.2.e0009

El contenido está bajo Licencia Creative Commons AtribuciónNoComercial-Compartir Igual 4.0 Internacional. Atribución - No Comercial - Compartir Igual (BY-NC-SA): no se permite un uso comercial de la obra original ni de las posibles obras derivadas, la distribución de las cuales se debe hacer con una licencia igual a la que regula la obra original.

\section{cc) (ㄱ) (-)}

Esta obra está bajo una Licencia Creative Commons Atribución. CompartirTgual 4.0 Internacional.
Resumen: Este artículo analiza el reclutamiento ministerial y los modos de hacer política que se consolidaron en las áreas de gestión socio-asistencial durante los gobiernos del PRO y de Cambiemos en tres niveles de gobierno distintos: Ciudad de Buenos Aires, provincia de Buenos Aires y el nivel nacional. A partir de un análisis multinivel que incluyó una base de datos con 151 trayectorias y entrevistas a 32 funcionarios, mostramos el pragmatismo que tuvo la nueva derecha argentina en el gobierno de lo socio-asistencial donde, entre 2007 y 2019 , desarrolló un estilo de gestión preocupado por la cercanía estatal y comprometido con el proyecto político del PRO y de la alianza Cambiemos en el marco de una heterogeneidad de propuestas formuladas para territorios con desafíos diversos.

Palabras clave: desarrollo social, reclutamiento político, Cambiemos, PRO, niveles de gobierno.

\begin{abstract}
This article analyzes the political recruitment and the ways of doing politics that were consolidated in the state social assistance during the PRO and Cambiemos goverments at three different levels of government: City of Buenos Aires, province of Buenos Aires and the level national. From a multi-level analysis that included a database with 151 trajectories and interviews with 32 officials, showed the pragmatism that the new Argentine right had in the social-welfare government where, between 2007 and 2019, they developed a style of social management concerned with the proximity of the state and committed to the political project of the PRO and the Cambiemos Alliance within the framework of a heterogeneity of proposals formulated for territories with diverse challenges.
\end{abstract}

Keywords: state social assistance, political recruitment, Cambiemos, PRO, Scales.

\section{Introducción}

La victoria de Mauricio Macri en el nivel nacional y de Cambiemos en distintas provincias y municipios de la Argentina en las elecciones de 2015 produjo un quiebre en el escenario político argentino signado por el ascenso de una nueva fuerza de derecha en distintos niveles de gobierno. ${ }^{[1]}$ Estas nuevas 
experiencias de gobierno se presentaron como una oportunidad política para que distintos individuos que no habían ocupado cargos públicos previamente realicen su primera experiencia de gestión estatal. Diferentes trabajos mostraron este proceso (Canelo y Castellani, 2016; Vommaro, 2017; entre otros), analizando el modo en que CEOs e individuos provenientes de distintas ONGs se presentaron como perfiles que asumieron un nuevo protagonismo en estos elencos de gestión gubernamental.

Sin embargo, el 2015 también apareció como una coyuntura favorable para que distintos funcionarios políticos del bastión electoral del PRO (la ciudad de Buenos Aires (CABA) migren hacia otros cargos de gobierno con desafíos y estructuras de gestión diferentes. La ocupación de cargos públicos nacionales, el arribo a la administración bonaerense o el ascenso en el organigrama de la ciudad de Buenos Aires se presentaron como trayectorias posibles gracias a esas nuevas victorias electorales.

En general, lo local, lo provincial y lo nacional fue estudiado de forma aislada y el modo en que esta nueva derecha decidió gobernar distintos niveles (y sus desafíos específicos) fue poco analizado. Los trabajos sobre esta nueva derecha se enfocaron en un nivel de gobierno (en principio la ciudad de Buenos Aires y luego, sobre todo, el nivel nacional) priorizando diferentes dimensiones como los discursos (Schuttenberg, 2017; Slimovich, 2014), las trayectorias (Canelo y Castellani, 2016; Vommaro, 2017) y las políticas (Medina, 2019; Rodríguez, 2017), entre otras.

En función de la novedad que implicaron las distintas victorias que obtuvieron el PRO y Cambiemos en 2015, analizamos el reclutamiento y el modo de hacer política que esta nueva derecha construyó en tres niveles de gobierno distintos: Nación, CABA y provincia de Buenos Aires (PBA) en un área de gestión en particular (Desarrollo Social). Como veremos a lo largo del artículo, indagamos las trayectorias de 151 funcionarios que ocuparon cargos públicos en los ministerios de Desarrollo Social mencionados, las características más relevantes de los escenarios institucionales que tuvieron que gobernar y los atributos de gestión que jerarquizaron en cada caso.

Realizamos un análisis de distintos niveles de gobierno en el marco de una serie de particularidades que caracterizan la dinámica política de las últimas décadas en la Argentina, atravesada por tres procesos que ponen en el centro de la discusión la cuestión de las escalas en el análisis político y que afectan particularmente las áreas asistenciales de gestión estatal.

El primero de ellos refiere a la territorialización de la acción política de los sectores populares (Merklen, 2010) y a la progresiva consolidación del área de Desarrollo Social como una agencia específica de gestión pública. Desde la vuelta de la democracia, buena parte de la organización y movilización colectiva popular tuvo como centro organizativo el barrio y se fue orientando al reclamo de políticas asistenciales. Como resultado de ello, se desarrolló una nueva institucionalidad vinculada a la asistencia en distintos niveles de gobierno destinada a intervenir en el creciente mundo del empleo informal y desocupado.

En segundo lugar, diferentes investigaciones discutieron la idea de Estado mínimo que prevaleció en algunas lecturas de la década de 1990 en la Argentina a partir del análisis de procesos que mostraron un rol activo del Estado en el marco de transformaciones importantes que modificaron su propia composición 
y protagonismo (Oszlak, 2003). Entre ellos, se destacó la subnacionalización del Estado argentino, que se inició a mediados de la década de 1970 y se profundizó en la década de 1990, a partir del cual los Estados provinciales y municipales asumieron responsabilidades vinculadas a áreas claves como la Educación y la Salud. En ese marco, un conjunto de trabajos comenzó a indagar las características de los Estados subnacionales, analizando dimensiones importantes como sus elites políticas, sus historias institucionales y sus políticas de gobierno (Ortiz de Rosas, 2016).

Por último, la victoria nacional del ex jefe de gobierno porteño Mauricio Macri en el año 2015 se presentó como una coyuntura favorable para seguir analizando el proceso de desnacionalización del sistema político argentino. Como muestran distintos trabajos, la desnacionalización de los partidos históricos de la argentina (UCR y PJ) se produjo en paralelo a una creciente autonomía de la política provincial (Leiras, 2007). Distintos partidos y dirigentes subnacionales aparecieron como actores claves del escenario político argentino $\mathrm{y}$, en algunos casos, realizaron alianzas exitosas de alcance nacional en el marco de distintas estrategias que incluyeron desde acuerdos entre una diversidad de gobernadores hasta prácticas que buscaron promover alianzas desde un bastión electoral hacia el resto del territorio (Cruz, 2018).

En el marco de un nuevo repertorio de acción colectiva de los sectores populares vinculado a su territorialización, de la importancia que asumieron los Estados subnacionales en áreas estratégicas de intervención social estatal y de un sistema político en el cual los partidos provinciales viene ganando autonomía, analizamos la gestión estatal asistencial de la nueva derecha argentina (PRO y Cambiemos) en tres niveles de gobierno distintos: Ciudad Autónoma de Buenos Aires, provincia de Buenos Aires y el nivel nacional.

Desde la sociología política, estudiamos esas escalas a partir del análisis de tres dimensiones (escenarios institucionales, trayectorias y atributos de gestión) que nos permitieron comprender el pragmatismo que caracterizó la gestión socioasistencial del PRO y de Cambiemos. En el primer apartado reflexionamos sobre los escenarios institucionales y, con ello, nos referimos a los contextos institucionales e históricos en los que se insertaron los funcionarios. En el segundo apartado, analizamos las trayectorias de quienes ocuparon cargos ministeriales que nos impulsaron a definir el tipo de reclutamiento que construyó esta nueva derecha como evitismo liberal. En el último apartado, estudiamos los atributos de gestión que jerarquizó esta alianza política para gobernar una parte importante de la cuestión social Argentina. Para ello, analizamos las concepciones y prácticas concretas que los funcionarios desarrollaron en el transcurso de la gestión, explicando desde una perspectiva comportamental de la elites (Cammack, 1990) lo que las mismas priorizan en determinadas situaciones. En función de esto último, construimos una tipología de funcionarios de esta nueva derecha en el área socio-asistencial.

Nuestro trabajo incluyó la utilización de diversas estrategias metodológicas. En principio, realizamos una breve sociohistoria de los ministerios indagando boletines oficiales y trabajos especializados, que nos permitieron reconstruir las historias de las carteras, sus estructuras organizativas y su papel en cada uno de los niveles de gobierno. Por otro lado, realizamos una base de datos con las 151 trayectorias educativas, sociales, políticas y profesionales de los 
individuos que ocuparon cargos públicos entre 2007 y 2017 en las carteras socioasistenciales del PRO y de Cambiemos. ${ }^{[2]}$ Por último, realizamos entrevistas a 32 funcionarios entre abril de 2016 y febrero de 2019, que no sólo nos permitieron reconstruir aspectos significativos de sus trayectorias sino que fueron relevantes para comprender dinámicas ministeriales, definiciones sobre las políticas del área y desafíos cotidianos de gestión. Además incorporamos entrevistas a 13 trabajadores estatales con el objetivo de contrastar la perspectiva de los funcionarios con la de quienes se encargaban de la implementación de las políticas socio-asistenciales. Todo ello fue analizado junto a notas periodísticas en las que interlocutores claves de las carteras (referentes sociales, otros funcionarios estatales, entre otros) alzaron su voz respecto a temas importantes de las agendas ministeriales.

\section{Escenarios institucionales en los distintos niveles de gobierno: problemas sociales relevantes, actores socio-políticos e historias ministeriales}

El análisis multinivel ocupó un rol clave para comprender la diversidad de desafíos que tuvieron que gestionar los cuadros sociales de la nueva derecha argentina. El escenario institucional es el ministerio en cada escala y, en ese sentido, analizamos tres dimensiones que fueron fundamentales para definir esos espacios diversos en los que se insertaron los funcionarios: los problemas sociales relevantes, los actores socio-políticos y las historias ministeriales. ${ }^{[3]}$ En este punto, sistematizamos un conjunto de trabajos y datos que mostraron: las estructuras sociales y económicas de cada territorio, actores que se presentan como interlocutores fundamentales del ministerio y las historias institucionales de cada cartera, prestando especial atención a la consolidación previa de épocas de gestión (Perelmiter, 2016).

En primer lugar, es importante mencionar algunas diferencias demográficas significativas de cada territorio: la Argentina tiene según el censo nacional de 2010 más de 40 millones de habitantes, de los cuales cerca de 3 millones viven en CABA y más de 15 millones en territorio bonaerense.

En relación a los problemas sociales que encontramos en cada nivel, observamos que desde 2002 la situación social a nivel nacional mostró mejoras en indicadores sensibles como la pobreza. ${ }^{[4]}$ Pese a esto último, los distritos elegidos muestran realidades diferenciadas: la ciudad de Buenos Aires es el distrito más rico del país pero cuenta con problemas importantes que se expresan en sus índices de pobreza e indigencia. ${ }^{[5]}$ Por su parte, la PBA presenta hace varias décadas números peores que $\mathrm{CABA}$ y el dato se agrava para el conurbano donde los indicadores aumentan. ${ }^{[6]}$

A partir de 2015, la coyuntura mostró otra complejidad producto del ajuste estructural que implementó Cambiemos desde su llegada al gobierno nacional, profundizado luego del acuerdo con el Fondo Monetario Internacional en 2018. La responsabilidad de la fuerza política respecto de las condiciones de vida de los sectores populares, afectadas desde la devaluación inicial, marcaron la agenda ministerial de las carteras de Desarrollo Social que debieron responder 
al aumento de índices como el de pobreza e indigencia. ${ }^{[7]}$ Por su parte, los indicadores de PBA y CABA también empeoraron. ${ }^{[8]}$

Otra dimensión constitutiva de la noción de escenario institucional refiere a los actores socio-políticos que se presentaron como articuladores relevantes de las políticas ministeriales. En el nivel nacional, el triunvirato de las organizaciones sociales (CCC, CTEP y Barrios de Pie) se consolidó durante la gestión de Cambiemos como un actor social fundamental del área. La capacidad de movilización que mostraron esas organizaciones en las últimas décadas y la jerarquización de referentes sociales en los cargos públicos de la cartera socioasistencial durante el kirchnerismo las ubicó como articuladores trascendentales. Esa acumulación histórica y el agravamiento de la crisis luego del 2018 sellaron su lugar estratégico.

En la provincia de Buenos Aires, y en el marco de una extendida hegemonía peronista, los 135 municipios que la integran y sus secretarios de las áreas socio-asistenciales aparecieron como interlocutores fundamentales. Pese a tener un restringido marco de autonomía jurídica en relación al gobierno provincial (Badia y Saudino, 2015), algunos municipios del conurbano bonaerense se han posicionado como actores políticos de peso provincial y nacional. En la ciudad de Buenos Aires, las victorias consecutivas que consiguió el PRO desde 2005 y la incapacidad de la oposición para referenciar un candidato propio marcaron una estabilidad sin precedentes en su corta pero convulsionada historia desde la sanción de la autonomía.

La última dimensión central de nuestra concepción de escenario institucional refiere a las historias ministeriales y los recursos de los que disponen los funcionarios de las carteras. Las áreas socio-asistenciales crecieron en coyunturas críticas como la década de 1990 y el post 2001. Como lo señalan distintas investigaciones (Giorgi, 2015; Grassi, 2006; Perelmiter, 2016), entre el propósito de afianzarse como un área técnica y el objetivo de desarrollar proximidad estatal con los sectores populares se fueron consolidando como áreas institucionales en distintos niveles de gobierno.

A partir de la estabilidad de algunos equipos ministeriales y sus políticas, diversos trabajos de ciencias sociales han identificado dos grandes épocas de gestión socio-asistencial desde la vuelta de la democracia hasta la llegada del macrismo. La primera de ellas es el modelo de la gerencia social y tiene como máximo exponente al secretario de Desarrollo Social del menemismo Eduardo Amadeo (Giorgi, 2015). En consonancia con los postulados de organismos internacionales como el Banco Mundial y el BID, se valorizó en la década de 1990 a nivel nacional e impactó de manera disímil en diferentes territorios subnacionales, ya sea a partir de la capacitación de sus funcionarios o por la formación de funcionarios nacionales que luego pasaron a ocupar cargos importantes locales o provinciales como los casos de Horacio Rodríguez Larreta y de María Eugenia Vidal en la ciudad de Buenos Aires. Este estilo (caracterizado por la ponderación de la eficiencia, la integralidad, la focalización y la descentralización) se consolidó en el Estado (y en otras instituciones como centros de expertise, organismos internacionales, ONGs, universidades) en un contexto de expansión de políticas de libre mercado, construyendo la legitimidad de sus prácticas a partir del saber experto y de la pretendida «objetividad» de los datos que construían sus referentes (Vommaro, 2011). 
El segundo modelo estuvo atravesado por la importancia de la figura de Eva Perón al interior de la tradición peronista y se destacó por su pretensión territorial (Barry, Ramaciotti y Valobra, 2008). Inspirados en la tradición evitista, desde la experiencia duhaldista que consolidó un imaginario tradicional de la mujer en política, vinculándola a las tareas del cuidado y de la supervivencia, hasta el estilo de gestión profesionalizado y militante de Alicia Kirchner, quien jerarquizó militantes de los movimientos sociales y trabajadores sociales, se buscaba recrear la promesa de proximidad estatal (Masson, 2004; Perelmiter, 2016). Como contracara de dos nociones centrales del modelo gerencial, la focalización de las políticas y la valorización del saber técnico, este tipo de gestión socio-asistencial buscó implementar políticas con cierto grado de universalidad fundando la legitimidad de sus prácticas en la cercanía entre Estado y ciudadanos.

En el marco de esa historia, cada uno de los ministerios se diferencia, además, por administrar recursos de diferente envergadura. A nivel presupuestario, el MDSN asumió una importancia creciente en el gabinete nacional desde el año 2003: del 2,56\% del presupuesto total en 2003 pasó a representar un 5,68\% en 2014. Por su parte, el personal total de la cartera pasó de 699 personas $(0,34 \% \mathrm{del}$ total) en 2003 a 3739 (0.99\% del total) en 2014 . El macrismo sostuvo números similares ${ }^{[9]}$ y profundizó el presupuesto de la cartera a partir de la incorporación de las políticas de salud, hábitat y seguridad social. El presupuesto ministerial de 2019 pasó a representar un 48,70\% del total ${ }^{[10]}$ y los empleados pasaron de 3740 en 2016 a 23506 en 2019. El salto porcentual en términos de empleados fue también significativo: pasó de tener casi el 1\% de los empleados nacionales al 6,5\% en 2019.

En PBA se fueron construyendo instituciones estatales provinciales destinadas a implementar políticas públicas asistenciales aunque, pese al hito que representó la gestión de «Chiche» Duhalde, no se fortaleció la cartera institucionalmente $y$, en los años siguientes, fue perdiendo recursos para invertir en políticas sociales y en cargos político-técnicos (Camou y Mateo, 2007). Desde la llegada de Cambiemos, el ministerio ocupó un lugar residual en relación al gasto total, llegando a un piso en el año 2016 de 2,37\%. En 2019, Desarrollo Social contrastaba con las agencias presupuestariamente más importantes como la Dirección General de Cultura y Educación y la cartera de Seguridad, quienes representaban un $23,94 \%$ y un $8,89 \%$, respectivamente, respecto del gasto total.

En la ciudad de Buenos Aires, entre 2007 y 2019 el ministerio mostró una tendencia presupuestaria decreciente (con un promedio de participación respecto del presupuesto total de un 5,15\%), mientras que mantuvo la cantidad de personal. En contraposición con la etapa previa (1997-2007), el porcentaje de gasto en el ministerio tuvo un descenso respecto al gasto total que se vio reflejado también en la meseta que muestra el número de trabajadores de la cartera (Salerno, 2018). Hacia el año 2019 Hábitat y Desarrollo Humano ocupaba el sexto lugar presupuestario entre los ministerios de CABA, con un declive que contrastaba con los presupuestos crecientes de los ministerios de Justicia y Seguridad $\left(3^{\circ}\right)$, Ambiente y Espacio Público $\left(4^{\circ}\right)$ y Desarrollo Urbano y Transporte $\left(5^{\circ}\right)$.

En términos nominales, las «cajas» son significativamente diferentes en cada nivel de gobierno: si tomamos el presupuesto designado en el año 2018 para 
cada ministerio observamos que, respecto del presupuesto nacional, el de CABA representa el 6,20\% y el de PBA el 9,28\%.

Sin embargo, consideramos fundamental pensar los ministerios desde una perspectiva que nos permita ir más allá de las partidas presupuestarias y sus estructuras organizativas. En ese sentido, y desde una mirada crítica de los enfoques institucionalistas, la noción de escenarios institucionales permite analizar los ministerios como espacios en construcción en los que se cruzan múltiples actores estatales y sociales. Los organigramas y las «cajas» de cada cartera son sólo una dimensión de esos espacios a gobernar que incluyen una diversidad de variables sociales y políticas que nosotros buscamos incorporar y diferenciar desde el primer momento de la investigación (y que seguirá presente a lo largo del análisis).

La noción de escenarios institucionales no sólo revela un conjunto de desafíos específicos vinculados a la gestión socio-asistencial desde una mirada preocupada por el vínculo entre lo político y lo social, sino que además se presenta como una herramienta para analizar las elites de gobierno en un estudio multinivel. Las construcciones históricas de esos escenarios se conforman en un entramado de actores y problemas que presentan particularidades específicas en cada nivel de gobierno aunque muestran algunas dimensiones comunes en un área estatal que interviene en las condiciones de vida de los sectores populares argentinos y que se ha venido consolidando dentro de distintos gabinetes.

\section{Reclutamiento político en el área socio-asistencial: el estudio de las trayectorias}

En paralelo al análisis de los escenarios institucionales, las trayectorias de los funcionarios ministeriales permiten comprender el reclutamiento político que tuvo cada cartera. La noción de trayectoria remite a «el recorrido biográfico individual en todas sus dimensiones, sin escindir la trayectoria política de la religiosa, de la laboral, etc., sino considerándola una unidad compleja, que va delineando perfiles individuales» (Giorgi, 2015: 21).

Esta nueva derecha no sólo mostró estrategias de reclutamiento diversas sino que individuos que fueron funcionarios en CABA y luego en PBA o en el nivel nacional resaltaron atributos distintos para presentarse a sí mismos en sus roles ministeriales según se tratase de un territorio u otro. Peronistas que ocuparon cargos públicos en CABA y saltaron a la provincia luego de la victoria de María Eugenia Vidal, mostraron concepciones críticas de las intermediaciones sociales entre el Estado y los ciudadanos al explicar su llegada al GCBA (en línea con las concepciones liberales que primaron en ese distrito), al tiempo que jerarquizaron saberes vinculados a la negociación con actores sociales y políticos para describir su recorrido hacia la PBA. A su vez, funcionarios provenientes de fundaciones que ocuparon cargos públicos en $\mathrm{CABA}$ y el nivel nacional luego de 2015, construyeron discursos que valorizaban los saberes de gestión privado para referirse a sus reclutamientos en el GCBA, al tiempo que rescataban su capacidad para la negociación con los movimientos sociales en el marco de la experiencia acumulada en la cartera porteña que los llevaba a ocupar cargos públicos en el nivel nacional. 
Sin embargo, las continuidades nos permitieron hablar de una época de gestión durante estos años que nació en la ciudad de Buenos Aires y que buscó replicarse en los distintos niveles de gobierno analizados. Como muestra Cruz (2018) al analizar las coaliciones por penetración estatal, el modelo de gestión social de esta nueva derecha partió de un bastión (CABA) hacia el resto del territorio. En línea con las investigaciones que analizan la desnacionalización del sistema político argentino y la autonomía (y relevancia) creciente de la política subnacional, comprender las características que tuvo esa experiencia de gestión local resulta central para analizar el modo en que esta nueva derecha gobernó un área estratégica en distintos niveles de gobierno.

Definimos como evitismo liberal el reclutamiento que realizó el PRO en la cartera porteña. Individuos provenientes de fundaciones cercanas a Larreta que participaron en la gestión pública en la década de 1990 y algunos dirigentes del peronismo porteño con años de militancia en los barrios del sur de la ciudad pasaron a ocupar un rol clave en el ministerio socio-asistencial de la ciudad de Buenos Aires. ${ }^{[11]}$ Este reclutamiento combinaba dos tradiciones bien diferentes como la liberal y la peronista, buscando importar saberes y destrezas disímiles como la expertise en políticas sociales y la negociación con organizaciones sociales, fundamentales para la construcción de gobernabilidad desde el área.

El evitismo liberal se replicó, con matices y tensiones, en la provincia de Buenos Aires y en el nivel nacional aunque se incorporaron dos espacios de reclutamiento: la política bonaerense y la alta gerencia empresarial. En todos los casos, los individuos con historia en el peronismo desempeñaron funciones claves del ministerio, como la negociación con los movimientos sociales y diversos actores político-institucionales. Carlos María Pedrini se destacó en el nivel nacional mientras que Santiago López Medrano fue elegido ministro durante todo el gobierno de Vidal. Ambos tenían historia en el peronismo y se incorporaron al GCBA luego de la victoria del PRO en 2007.

Por su parte, dirigentes partidarios, entre los que se destacaron los propios cuadros del PRO, fueron ocupando roles cada vez más relevantes. El lugar central que tuvo la ministra Stanley en las negociaciones con los movimientos sociales en el nivel nacional fue expresión de esto último y mostró diferencias sustanciales con sus propias prácticas en la ciudad de Buenos Aires, donde los peronistas habían sido quienes sellaron el vínculo con las organizaciones sociales. Pese a ser reclutados en sus inicios por sus trayectorias «sociales», se politizaron en la ciudad de Buenos Aires luego de 10 años de gestión local. Incorporaron allí destrezas vinculadas a tareas comprometidas con los objetivos políticos de la alianza gobernante, ya sea relacionadas con la negociación política o con la preocupación por la dimensión electoral, que les sirvieron más tarde para conducir la cartera nacional. A su vez, la experiencia en el gobierno de la ciudad de Buenos Aires implicó, luego de las victorias de 2015, la llegada a los cargos públicos de las carteras socio-asistenciales de CABA, PBA y el nivel nacional de muchos funcionarios con más de 8 años de experiencia en el sector público lo que diferenció a la derecha argentina de otras experiencias latinoamericanas como la de Temer en Brasil, donde el reclutamiento del gabinete se ancló en individuos con trayectorias públicas pero provenientes del ámbito legislativo, o la de Pedro Pablo Kuczynski en Perú caracterizada por la llegada de individuos 
con trayectorias mayoritariamente público-privadas o privadas puras (Nercesian y Cassaglia, 2019).

El reclutamiento de los tres ministerios socio-asistenciales replicó, en sus trazos gruesos, el de la ciudad de Buenos Aires aunque se redefinieron algunas estrategias en función de los desafíos y los objetivos que se plantearon en cada caso. En el nivel nacional la importancia que asumieron los CEOs en el armado del gabinete se expresó también en el área social ${ }^{[12]}$, donde, sin ser mayoría, pasaron a ocupar cargos públicos un conjunto de individuos cercanos a Mario Quintana ${ }^{[13]}$. Por su parte, en la provincia de Buenos Aires, de extensa tradición peronista y con una multiplicidad de municipios, Cambiemos jerarquizó el trabajo de dirigentes con trayectoria en la política provincial posicionando en los cargos de mayor rango a un conjunto de individuos que disputaban distritos del conurbano bonaerense ${ }^{[14]}$. Desde el trabajo en la cartera, estos últimos priorizaron la llegada a los territorios que disputaban y favorecieron la construcción de un perfil cercano, preocupado por los problemas que enfrentan esos municipios.

El análisis en distintas escalas mostró las características principales que definieron el reclutamiento que construyó esta nueva derecha en el área socioasistencial y el modo en el que los desafíos diversos que se presentaron en esos territorios fueron abordados. Se incorporaron individuos con experiencia en la política bonaerense en la cartera provincial y en la alta gestión empresarial en la gestión nacional, aunque en ambos reclutamientos jugaron un rol central algunos funcionarios con historia en el peronismo y funcionarias cercanas a Larreta (formadas en sus fundaciones) respetando los lineamientos generales que se afianzaron luego de 8 años de gobierno porteño.

$\mathrm{El}$ análisis de las trayectorias de los funcionarios no sólo mostró los espacios sociales y políticos de los cuales se nutrieron el PRO y Cambiemos para gestionar un área sensible como Desarrollo Social sino que, en el marco de un análisis multinivel, reveló el pragmatismo y la diversidad de estrategias que construyó esta nueva derecha para gobernar niveles de gobierno con desafíos y estructuras muy distintos.

Además, y como veremos en el apartado siguiente, las variables sociales, políticas y económicas presentes en las biografías de los individuos se presentan como dimensiones centrales para comprender el modo en que se hizo política al interior del área en estos años de gestión socio-asistencial.

\section{Atributos de gestión y perfiles de funcionarios sociales del PRO y Cambiemos}

Para analizar los modos de hacer política al interior de los ministerios, estudiamos los atributos de gestión que ponderó esta nueva derecha en el área socioasistencial, entendiendo por ellos estilos de trabajo, de hacer y de poner en escena decisiones, que representaron valores y concepciones que caracterizaron las gestiones del PRO y de Cambiemos. Los atributos son el resultado del cruce entre las trayectorias de los funcionarios, los desafíos que presentó la gestión de Desarrollo Social en cada territorio y aquello que realizaban práctica y discursivamente en el devenir de la gestión estatal. Como bien muestran distintos trabajos que analizan élites ministeriales (Gené, 2019; Perelmiter, 2016), las 
relaciones y los capitales que permiten el ingreso a un cargo público se ponen en juego en el desarrollo de la gestión en función de los conflictos y objetivos que enfrentan los funcionarios. En esa intersección, los resultados posibles pueden ser disímiles: se consolidan saberes previos, se desarrollan destrezas nuevas, se construyen lealtades diferentes.

Los atributos son categorías no excluyentes que se combinan entre sí y que describen el conjunto de concepciones y prácticas que se ponderaron en el área socio-asistencial. En total, son siete: 1) lealtad personal con figuras importantes de la coalición sociopolítica, 2) saber de gestión, 3) manejo de procesos administrativos públicos, 4) capacidad de negociación con organizaciones de distinto tipo, 5) búsqueda de cercanía con trabajadores del área y con los destinatarios de las políticas, 6) compromiso con el proyecto político, y 7) la condición de mujer.

A su vez, en el marco de un estudio multinivel nos preguntamos: ¿cómo repercuten los distintos escenarios institucionales en el análisis de los atributos de gestión jerarquizados? Como veremos más adelante, las escalas no sólo nos mostraron la heterogeneidad de prácticas y discursos sino un conjunto de estrategias construidas por los funcionarios en respuesta a los desafíos de gobierno que se presentan en cada situación.

Los funcionarios construyeron una fórmula propia que se replicó en las distintas carteras aunque asumió particularidades específicas en cada caso, acentuando rasgos e innovando en los distintos niveles de gobierno. La estabilidad lograda tras 8 años de gobierno en la ciudad de Buenos Aires le permitió al PRO consolidar una época de gestión caracterizada por la jerarquización de un saber técnico vinculado a la gestión socio-asistencial adquirido por un conjunto de individuos formados en fundaciones y con experiencia en el área en la década de 1990, a lo que se sumó un nuevo compromiso con el proyecto político del PRO y la pretensión de construir prácticas y discursos cercanos con los destinatarios de las políticas sociales.

Esa fórmula propia del PRO se trasladó a los nuevos niveles de gobierno analizados, donde Cambiemos innovó en función de los desafíos que presentaron esos territorios. En la PBA, un conjunto de candidatos de Cambiemos en distintos municipios del conurbano bonaerense buscaron construir cercanía con la ciudadanía de esos distritos. Por su parte, CEOs e individuos provenientes de fundaciones ligadas al empresariado jerarquizaron un saber de gestión en el nivel nacional, ya no vinculado a los programas sociales, sino a un conjunto de habilidades manageriales orientadas a la capacidad de instrumentar decisiones estratégicas $^{[15]}$.

Definimos a estos cuadros sociales como una nueva derecha embarrada en virtud de una premisa que se jerarquizó en el área y que implicó en estos años generar prácticas y discursos que permitieran reducir la distancia entre las autoridades ministeriales y el resto de los trabajadores y la ciudadanía. Recuperando la preocupación histórica del área y las estrategias más generales del PRO como partido político, los funcionarios más importantes fueron aquellos que comprendieron la relevancia de esa dimensión para legitimar las prácticas políticas.

El paso por los ministerios aparecía en la mayoría de los casos como una instancia clave para reconstruir las lealtades que permitieron el ingreso al armado 
PRO. Con la palabra lealtad nos referimos a un tipo de confianza interpersonal que toma la forma de un vínculo directo entre un jefe político que administra recursos y define las reglas del grupo, y un conjunto de individuos que le prestan reconocimiento a cambio de gratificaciones -simbólicas, identitarias, materiales(Giorgi, 2015).

Las relaciones y trayectorias que resultaron fundamentales para comprender los ingresos de los funcionarios al ministerio se resignificaron a medida que se fueron construyendo dinámicas nuevas al interior de la cartera y de la coalición de gobierno. Muchos funcionarios que trabajaron con Vidal en CABA generaron una nueva lealtad con la ex ministra a partir de un trabajo específico en la gestión porteña que incluía reuniones de gestión hasta campañas políticas que la tenían como protagonista (vice jefatura de gobierno en 2011 y gobernación de la provincia de Buenos Aires en 2015). A partir de ello, decidieron pasar a la gestión bonaerense (en ruptura con lealtades previas) apostando por el futuro político de una referente que ganaba poder al interior de la alianza.

Por otro lado, en tiempos de críticas a los políticos ${ }^{[16]}$, los funcionarios hicieron política a partir de la ponderación de la «gestión» como valor en sí mismo. Con todo, la valorización de esto último supuso prácticas diferentes en los niveles de gobierno analizados. En la ciudad de Buenos Aires, la llegada de funcionarios expertos formados en fundaciones que pasaron por la gestión pública en tiempos de menemismo y aliancismo, parecía replicar un modelo eficientista que primó en la década de 1990. La especialización técnica de los funcionarios más importantes, formados en programas de transferencia condicionada de ingresos, los vinculaba a los expertos que participaron en las últimas décadas del siglo XX del nuevo campo de «lucha contra la pobreza» (Vommaro, 2011). En la provincia la idea de orden tuvo mucha fuerza luego de 30 años de gobiernos peronistas y el «saber de gestión» se vinculó a las destrezas de dos grupos: en primer lugar, un conjunto de individuos provenientes del sector privado que ocuparon cargos públicos en el área de Deportes y, en segundo lugar, funcionarios provenientes de la gestión pública porteña que importaron el modelo de gestión socio-asistencial allí implementado. En el nivel nacional, la valorización del saber de gestión fue abordada desde un estilo managerial que se construyó como respuesta al modelo territorial del kirchnerismo y a las críticas que distintos actores sociales y políticos venían realizando a las «excesivas» intermediaciones sociales en las relaciones entre Estado y sectores populares. Sin embargo, como destacaron distintos autores (Hudson, 2017; Longa, 2019), los programas que fueron emblema del kirchnerismo tuvieron continuidad y los movimientos sociales siguieron jugando un rol relevante en la llegada de las políticas al territorio.

En la ciudad de Buenos Aires encontramos una particularidad: la migración de funcionarios hacia el nivel nacional y el provincial después de las victorias del 2015 marcaron la jerarquización de un particular saber de gestión: el manejo de procesos administrativos estatales. Se valorizaron, sobre todo luego del 2015, trayectorias burocráticas al interior del ministerio formadas en los años previos y dispuestas a ocupar cargos públicos a partir de la afinidad política con funcionarios que ascendían en las jerarquías de gobierno.

Por otro lado, y aunque parezca contradictorio con el propio discurso individualizante del macrismo (Canelo, 2019), todos los ministerios tuvieron 
alas políticas que marcaron las agendas ministeriales y la negociación con los actores sociales y políticos de peso que se presentan como interlocutores claves del área en cada nivel de gobierno.

En Nación, los funcionarios más importantes buscaron construir gobernabilidad generando canales de diálogo con el triunvirato de las organizaciones sociales en tiempos de empobrecimiento social. El secretario de Gestión y Articulación Institucional del MDSN, Carlos María Pedrini, con historia en el peronismo, y la propia ministra Stanley se consolidaron como figuras importantes, en parte, por su capacidad para negociar con esos actores.

En la provincia de Buenos Aires, la negociación estuvo centrada en la articulación con los 135 intendentes (y sus funcionarios socio-asistenciales) y con organizaciones de envergadura y presencia histórica en territorio bonaerense. A diferencia de la ciudad de Buenos Aires, donde la articulación tuvo que ver con algunas organizaciones pequeñas con las que se gestionaron políticas como los Centros de Primera Infancia, los funcionarios bonaerenses mostraron una heterogeneidad de destrezas para negociar con intendentes políticos del interior y del conurbano, al tiempo que disputaron algunos municipios en los que se presentaban como candidatos.

En todos los niveles de gobierno, la pregunta por la proximidad estatal nos permitió comprender el proceso de politización que atravesaron muchos funcionarios que habían ingresado al Estado como técnicos o a partir de sus perfiles «sociales». En el ejercicio de la actividad política en los años estudiados algunos de esos cuadros se comprometieron con los objetivos políticos del partido, ordenándose en líneas políticas internas y desarrollando destrezas asociadas a perfiles «políticos».

La cercanía entre autoridades estatales y el resto de la ciudadanía se presentó como un eje de las carteras, con distintas intensidades y prácticas en función de los territorios analizados. Este atributo, por el cual nominamos a esta derecha como embarrada, incluyó distintas dimensiones. Al interior de los ministerios, individuos procedentes de fundaciones o del mundo empresario buscaron en todos los niveles de gobierno importar los liderazgos manageriales valorizados en la vida cotidiana de las grandes empresas (Luci, 2016). Hacia afuera del ministerio, esos cuadros buscaron generar prácticas, como las visitas a comedores o los timbreos, con el objetivo de construir cercanía con los destinatarios de las políticas sociales. En el nivel nacional, la cercanía se escenificó en las redes sociales a partir de la presencia de la ministra en comedores de distintas ciudades del país. En territorio bonaerense, los candidatos priorizaron la llegada directa a los municipios que disputaban electoralmente, participando incluso de la implementación de políticas y obras de otros ministerios. Por último, en la ciudad de Buenos Aires, los funcionarios se involucraron en distintos momentos de la implementación de las políticas en el territorio, constituyendo un hito el Operativo Frío (Salerno, 2018).

Una buena parte de los cuadros «sociales» se politizaron en el transcurso de la gestión desarrollando con el tiempo perfiles cercanos y sensibles afines a una nueva democracia de proximidad (Annunziata, 2012). En sintonía con la estrategia general del PRO y de Cambiemos de generar cuadros de gestión y cercanos (atributos especialmente compatibles en tiempos de redes sociales), la afinidad electiva que encontramos entre esta histórica preocupación del área y esa 
dimensión universal que prima en este contexto democrático posterior al 2001 se presentaron como claves para comprender el compromiso político que muchos de estos individuos asumieron con esta nueva derecha y las líneas internas de cada uno de los ministerios analizados.

Por último, los funcionarios entrevistados mostraron que, al igual que en otras experiencias históricas (Masson, 2004), la sensibilidad social y la entrega por los necesitados se construyeron como dimensiones retóricas imprescindibles de una política llevada adelante por mujeres. La paradoja es evidente: la crítica a los políticos tradicionales se realizó a partir de la recreación de una fórmula tradicional de género y de un modo tradicional de comprender la asistencia social.

La consagración de una tradicional perspectiva de lo doméstico en la esfera pública fue el trampolín para que un conjunto de mujeres ocupara cargos relevantes en gabinetes de distintos niveles de gobierno, acumulando un poder político que, en diferentes circunstancias, las puso por encima de sus padrinos. Desde ese perfil, construyeron autonomía y poder de decisión propios posicionándose en áreas y jerarquías históricamente ocupadas por hombres, como rangos de ministro, y, en el caso paradigmático de Vidal, la gobernación de la provincia de Buenos Aires.

El territorio bonaerense fue el único distrito que no tuvo una ministra: Santiago López Medrano, con historia en el peronismo de San Martín, ocupó el máximo cargo de la cartera en función de su historia de trabajo con Vidal en CABA, de sus pretensiones electorales en un municipio y de un armado provincial en el cual sólo el $9 \%$ de las candidatas de Cambiemos a intendentes en 2019 eran mujeres (Salerno, 2020).

A partir del análisis de los atributos jerarquizados en estos años, formulamos una tipología de funcionarios del área socio-asistencial durante las gestiones del PRO y de Cambiemos (Cuadro 1). 


\section{Cuadro 1}

Tipología de funcionarios del área socio-asistencial PRO y de Cambiemos

\begin{tabular}{|c|c|}
\hline Perfiles de gestión & \multicolumn{1}{c|}{ Atributos } \\
\hline PRO puras & $\begin{array}{l}\text { 1) lealtad personal con figuras importantes de la coalición } \\
\text { sociopolítica, 2) expertise en la gestión de políticas socio- } \\
\text { asistenciales, 3) búsqueda de cercanía con trabajadores del } \\
\text { área y con los destinatarios de las políticas, 4) compromiso } \\
\text { con el proyecto político PRO, 5) la condición de Mujer. }\end{array}$ \\
\hline Expertos & $\begin{array}{l}\text { 1) expertise en la gestión de políticas socio-asistenciales, } \\
\text { 2) lealtad personal con figuras importantes de la coalición } \\
\text { sociopolítica. }\end{array}$ \\
\hline Conversos & $\begin{array}{l}\text { 1) lealtad personal con figuras importantes de la coalición } \\
\text { sociopolítica, 2) capacidad de negociación con organizaciones } \\
\text { de distinto tipo, 3) búsqueda de cercanía con trabajadores del } \\
\text { área y con los destinatarios de las políticas, 4) compromiso } \\
\text { con el proyecto político. }\end{array}$ \\
\hline Burócratas & $\begin{array}{l}\text { 1) lealtad personal con figuras importantes de la coalición } \\
\text { sociopolítica, 2) manejo de procedimientos político- } \\
\text { administrativos. }\end{array}$ \\
\hline Empresarios & $\begin{array}{l}\text { 1) lealtad personal con figuras importantes de la coalición } \\
\text { sociopolítica, 2) expertise en la gestión. }\end{array}$ \\
\hline Candidatos & $\begin{array}{l}\text { 1) lealtad personal con figuras importantes de la coalición } \\
\text { sociopolítica, 2) compromiso con el proyecto político, 3) } \\
\text { Cercanía con los destinatarios de las politicas, 4) capacidad } \\
\text { de negociación con organizaciones de distinto tipo. }\end{array}$ \\
\hline
\end{tabular}

Fuente: base de datos de elaboración propia.

Estudiar distintos niveles de gobierno nos permitió analizar el modo en que distintos atributos se reconfiguraban al tener que adaptarse a nuevos escenarios institucionales. En la ciudad de Buenos Aires, las PRO puras, que habían ingresado como técnicas al Estado en experiencias previas de gestión social, se posicionaron como las funcionarias más relevantes del área y se caracterizaron por la búsqueda de construir proximidad con el resto de la ciudadanía. La politización de esos cuadros «sociales» se expresó luego en victorias electorales que las tuvieron como protagonistas al tiempo que sus decisiones fueron muy relevantes para comprender las trayectorias del resto de los funcionarios.

En la provincia de Buenos Aires, la llegada de Cambiemos al Ministerio de Desarrollo Social repitió la ponderación de atributos de gestión porteños, a los que se agregaron recursos vinculados al conocimiento del territorio bonaerense y a la experiencia previa de gestión en altos cargos empresariales. Los candidatos asumieron un rol trascendental en una provincia con 135 municipios y con una buena parte de las intendencias gobernadas por el peronismo. Vidal decidió profundizar el vínculo entre el área socio-asistencial y lo político-electoral, del que ella misma era expresión, colocando a un conjunto de políticos bonaerenses 
en los cargos de alto rango ministerial desde donde se propusieron destronar al peronismo de distritos del Gran Buenos Aires.

En el nivel nacional, la particularidad estuvo dada por dos dimensiones centrales: la importancia que asumieron los funcionarios que venían del GCBA como ala política del ministerio y la relevancia cuantitativa que adquirieron los empresarios. Estos últimos estuvieron ausentes en la cartera porteña y se presentaron de manera marginal en el nivel provincial pero fueron singularmente importantes en el ministerio nacional en el marco de un gobierno en el cual los CEOs ocuparon casi un tercio de los cargos públicos del conjunto del gabinete (Canelo y Castellani, 2016).

\section{Reflexiones finales}

Pese a las derrotas de Mauricio Macri y de María Eugenia Vidal en 2019, consideramos fundamental estudiar las experiencias de gobierno de una nueva derecha que parece afianzarse como una protagonista central del escenario político argentino. Desde la sociología política y en el marco de un estudio multinivel, mostramos las características que definieron a sus cuadros sociales de gestión estatal al tiempo que destacamos su pragmatismo.

Este último no significó que no pudiéramos observar concepciones y prácticas de gobierno ancladas en ideologías que ubican a esta fuerza a la derecha del espectro político (el abandono de las políticas de DDHH en la ciudad de Buenos Aires fue un ejemplo en ese sentido) sino que tuvo que ver con la flexibilidad que mostró esta nueva derecha y sus funcionarios en relación a los medios y las prácticas implementadas para afrontar desafíos diversos.

Anclados en la sociología política abordamos el cruce de tres dimensiones que consideramos fundamentales para el análisis del trabajo político en la Argentina actual: los escenarios institucionales, las trayectorias y los atributos de gestión. Estos niveles los distinguimos analíticamente pero se superponen continuamente y su análisis conjunto forma parte del trabajo artesanal que buscamos reconstruir para explicar lo que caracteriza a nuestras élites políticas y los modos diversos en que se ejecuta el trabajo político en la democracia argentina.

La noción de escenario institucional mostró la diversidad de desafíos que enfrentan quienes asumen la tarea de gobernar, no sólo en relación a áreas específicas de gestión estatal sino a los problemas y actores que suponen diferentes niveles de gobierno. Las trayectorias nos permitieron analizar los mundos sociales de la política y las estrategias heterogéneas con las que esta fuerza política enfrentó el gobierno de lo social en territorios disímiles. El dogmatismo característico de otros partidos históricos de la derecha argentina, como la UCeDé, dio lugar a un conjunto diversos de perfiles en el área de Desarrollo Social, donde se destacó en el reclutamiento la convivencia de dos espacios bien distintos como las fundaciones y el peronismo.

Por último, el estudio de los atributos de gestión reveló un conjunto de discursos y prácticas que se elaboraron en carteras con historias particulares. El pragmatismo caracterizó estos años de victorias electorales: algunos peronistas individualizaron y «despolitizaron» sus discursos al ingresar a la gestión socioasistencial de la ciudad de Buenos Aires, repolitizándolos luego de la victoria de María Eugenia Vidal en la provincia y de la estrategia de disputar municipios 
del conurbano bonaerense desde el ministerio que estudiamos; los managers y los voluntarios, pese a presentarse como gestores más que políticos, empezaron a construir poder político disputando cargos y recursos desde un discurso técnico y despolitizado a partir del cual buscaron valorizar su trabajo; funcionarios nacionales que denunciaban las intermediaciones sociales negociaron con los movimientos sociales medidas importantes, como la Ley de Emergencia Social, en tiempos de empobrecimiento creciente mostrando una capacidad particular para compatibilizar esa práctica con la construcción de un perfil cercano, sin mediaciones y en contacto directo con la ciudadanía construido desde las redes sociales.

El estudio en distintas escalas nos permitió comprender las variaciones en la ejecución del trabajo político (Offerlé, 2011), ancladas en los desafíos heterogéneos que presenta cada nivel de gobierno en relación a interlocutores y problemas específicos. Seguir pensando a esta nueva derecha desde una propuesta analítica preocupada por mostrar la versatilidad de los procesos políticos es una tarea esencial de las ciencias sociales.

\section{Referencias}

ALENDA, STÉPHANIE (2020): «Introducción», en: ALENDA, STÉPHANIE (Ed.). Anatomia de la derecha chilena: Estado, mercado y valores en tiempos de cambio, Santiago de Chile, FCE-UAB, pp. 15-39.

ANNUNZIATA, ROCÍO (2012): « ¿Hacia un nuevo modelo de lazo representativo? La representación de proximidad en las campañas electorales de 2009 y 2011 en Argentina», en: CHERESKY, ISIDORO; ANNUNZIATA, ROCÍO (Comps.). Sin programa, sin promesa. Liderazgos y procesos electorales en Argentina, Buenos Aires, Prometeo, pp. 24-55.

BADIA, GUSTAVO; SAUDINO, MARTINA (2015): «La construcción políticoadministrativa del conurbano bonaerense», en: KESSLER, GABRIEL (Dir.). El Gran Buenos Aires. Historia de la provincia de Buenos Aires, Buenos Aires, Editorial Universitaria, Edhasa, pp. 103-128.

BARRY, CAROLINA; RAMACIOTTI, KARINA; VALOBRA, ADRIANA (2008): La Fundación Eva Perón y las mujeres: entre la provocación y la inclusión, Buenos Aires, Biblos.

CAMMACK, PAUL (1990): «A critical assessment of the new elite paradigm», en: American Sociological Review, 55, pp. 415-420.

CAMOU, ANTONIO; MATEO, SEBASTIÁN (2007): «¿El tiempo vence a la organización? Dinámica política, estructuras estatales y políticas sociales en la provincia de Buenos Aires (1984-2004)», en: Cuestiones de Sociología, 4, pp. 129-142.

CANELO, PAULA; CASTELLANI, ANA (2016): «¿El imperio de los CEOs? Una radiografía del primer gabinete nacional del gobierno de Macri», en: LIJALAD, ARIEL (comp.). Plan Macri. Argentina gobernada por las corporaciones, Buenos Aires, Peña Lillo-Ediciones Continente, pp. 129-136.

CANELO, PAULA (2019): ¿Cambiamos? La batalla cultural por el sentido común de los argentinos, Buenos Aires, Siglo XXI Editores.

CRUZ, FACUNDO (2018): Construyendo House of cards. Partidos y coaliciones en Argentina, 1995-2015, Ciencia Política (Doctorado), Escuela de Política y Gobierno, Universidad Nacional de San Martín. 
GENÉ, MARIANA (2019): La rosca politica. El oficio de los armadores delante y detrás de escena (o el discreto encanto del toma y daca), Buenos Aires, Siglo XXI editores.

GIORGI, GUIDO (2015): Modos de acceso y circulación por el gobierno nacional. Perfiles, sociabilidades y redes socio-politicas y religiosas de los cuadros de gobierno de Desarrollo Social de la Nación. Argentina, 1994-2011, Ciencias Sociales (Doctorado), Facultad de Ciencias Sociales de la Universidad de Buenos Aires École des Hautes Études en Sciences Sociales.

GRASSI, ESTELA (2006): Politicas y problemas sociales en la sociedad neoliberal, Buenos Aires, Espacio Editorial.

HUDSON, JUAN PABLO (2017): «Continuidad en los parches», en: Revista Crisis. Disponible en https://revistacrisis.com.ar/notas/continuidad-en-los-parches.

LEIRAS, MARCELO (2007): Todos los caballos del rey: la integración de los partidos políticos y el gobierno democrático de la Argentina, 1995-2003, Buenos Aires, Prometeo libros.

LONGA, FRANCISCO (2019): Historia del Movimiento Evita: La organización social que entró al Estado sin abandonar la calle, Buenos Aires, Siglo XXI Editores.

LUCI, FLORENCIA (2016): La era de los managers. Hacer carrera en las grandes empresas del país, Buenos Aires, Editorial Paidós.

MASSON, LAURA (2004): La politica en femenino. Género y poder en la provincia de Buenos Aires, Buenos Aires, Editorial Antropofagia.

MEDINA, LETICIA (2019): «De trabajadores a «costo laboral». Las políticas laborales en la era macrista», en: NAZARENO, MARCELO; SEGURA, MARÍA SOLEDAD; VÁZQUEZ, GUILLERMO (eds.). Pasaron Cosas. Politica y politicas públicas en el gobierno de Cambiemos, Córdoba, Editorial Brujas, pp. 221-244.

MERKLEN, DENIS (2010): Pobres ciudadanos. Las clases populares en la era democrática (Argentina 1983-2003), Buenos Aires, Editorial Gorla.

MORRESI, SERGIO; VOMMARO, GABRIEL (org.) (2015): Hagamos equipo. PRO y la construcción de una nueva derecha en Argentina, Buenos Aires, Universidad Nacional de General Sarmiento.

NERCESIAN, INÉS; CASSAGLIA, ROBERTO (2019): «Radiografía de los gabinetes ministeriales en Brasil y Perú (2016-2018). Un análisis comparativo», en: Telos, 2 (21), pp. 372-400.

OFFERLÉ, MICHEL (2011): «Los oficios, la profesión y la vocación de la política», en: PolHis, 7, pp. 84-100.

ORTIZ DE ROSAS, VICTORIA (2016): «La democracia Argentina en clave subnacional: nuevos objetos de estudio e interpretaciones sobre el Estado, los partidos políticos y las elites políticas», en: MAURO, SEBASTIÁN; ORTIZ DE ROSAS, VICTORIA; PARATZ VACA NARVAJA, MARTÍN (Comps). Politica Subnacional en Argentina. Enfoques y problemas, Buenos Aires, Facultad de Ciencias Sociales - Universidad de Buenos Aires, pp. 30-53.

OSUNA, MARÍA FLORENCIA (2016): La intervención social del Estado argentino entre dos dictaduras. Un estudio de los proyectos, las politicas y los actores del Ministerio de Bienestar Social/Acción Social de la Nación (1966-1983) (Doctorado), Facultad de Filosofía y Letras, Universidad de Buenos Aires.

OSZLAK, OSCAR (2003): «El mito del Estado mínimo: una década de reforma estatal en la Argentina», en: Desarrollo Económico, 168, volumen 42, pp.519-543.

PERELMITER, LUISINA (2016): Burocracia Plebeya. La trastienda de la asistencia social en el Estado Argentino, Buenos Aires, UNSAM Edita. 
RODRÍGUEZ, GINA PAOLA (2019): «Vueltas y revueltas de la derecha peruana en el Siglo XXI», en: Estudios Sociales del Estado, 9, volumen 5, pp. 24-54.

RODRÍGUEZ, LAURA GRACIELA (2017): «Cambiemos: la política educativa del macrismo», en: Question/Cuestión, 1, pp. 89-108.

SALERNO, AGUSTÍN (2018): Las que mandan. Perfiles y trayectorias del alto funcionariado del Ministerio de Desarrollo Social de la ciudad de Buenos Aires durante la gestión PRO (2007-2015), Ciencia Política (Maestría), Escuela Interdisciplinaria de Altos Estudios Sociales - Universidad Nacional de San Martín.

SALERNO, AGUSTÍN (2020): La cara social de la nueva derecha argentina. Perfiles políticos y atributos de gestión socio-asistencial del PRO y de Cambiemos en la Nación, la provincia de Buenos Aires y la ciudad de Buenos Aires (2007-2019), Sociología (Doctorado), Escuela Interdisciplinaria de Altos Estudios Sociales Universidad Nacional de San Martín.

SCHUTTENBERG, MAURICIO (2017): «La política de la despolitización. Un análisis de la construcción del relato PRO», en: Desafíos, 29, pp. 277-311.

SLIMOVICH, ANA (2014): «El discurso macrista en twitter: un análisis sobre la campaña para la reelección del jefe de gobierno de Buenos Aires», en: Revista de Estudios Politicos y Estratégicos, 2, volumen 1, pp. 8-27.

VOMMARO, GABRIEL (2011): «Los pobres y la pobreza como dominio experto: contribuciones a una socio-historia», en: Morresi, Sergio y Vommaro, Gabriel (Comps.). Saber lo que se hace. Expertos y politica en Argentina, Buenos Aires, Universidad Nacional de General Sarmiento-Prometeo, pp. 79-134.

VOMMARO, GABRIEL (2017): La larga marcha de Cambiemos. La construcción silenciosa de un proyecto de poder, Buenos Aires, Siglo XXI Editores.

ZACK, GUIDO; SCHTEINGART, DANIEL; FAVATA, FEDERICO (2020): «Pobreza e indigencia en Argentina: construcción de una serie completa y metodológicamente homogénea», en: Sociedad y economía, 40, pp. 69-98.

\section{Notas}

[1] Retomamos la definición que realizan Morresi y Vommaro (2015) para referirse al PRO y que luego retoma Vommaro (2017) para analizar a Cambiemos. Los autores utilizan el esquema de Pierre Ostiguy del doble espectro político argentino, o sea, junto al eje izquierda-derecha, incorporan lo alto (elitista y republicano) y lo bajo (como lo popular). En ese marco, ubican al PRO del centro a la derecha debido a sus relaciones con las redes de la derecha internacional pero también producto de su interés por ocupar el lugar más alto. Además, diferencian a esta nueva propuesta política, más pragmática según los autores, de experiencias previas como la UCeDé o partidos conservadores provinciales como el bloquismo en San Juan, que no pudieron proyectarse más allá de la conquista de bancas legislativas o de la gestión de gobiernos subnacionales.

[2] La decisión de incorporar las políticas de Salud y de seguridad social en el Ministerio de Desarrollo Social de la Nación (MDSN) a partir del año 2018 nos impulsó a realizar un corte en diciembre de 2017. De esta manera, elegimos una muestra de individuos vinculada al área socio-asistencial en los tres niveles de gobierno. Pese a que la base llega hasta 2017, el análisis de dinámicas ministeriales y conflictos que tuvieron como escenario estas tres carteras finaliza en 2019.

[3] Es importante destacar que el trabajo abordó temporalidades diversas: mientras que el ministerio porteño fue estudiado desde 2007, los ministerios de PBA y Nación fueron analizados entre 2015 y 2019. En este sentido, el desafío no fue sólo comprender las temporalidades locales y provinciales como algo distinto de las nacionales sino incorporar esta dimensión que afectó distintos aspectos relevantes analizados. 
[4] A raíz de la dificultad en la comparación entre períodos, agradecemos el aporte de Daniel Schteingart quien se propuso homogeneizar las canastas con el propósito de reconstruir las series en el largo plazo. El investigador establece que en 2002 se alcanzó un pico de 66\% en los niveles de pobreza, mientras que en 2007 se ubicó en 37,4\%.

[5] Desde la tasa de desocupación pico del 2001 (de casi un 20\% de la población económicamente activa), hasta los índices de pobreza (11,2\%, EPH-INDEC) e indigencia (2,2\%, EPH-INDEC) del 2018, la ciudad de Buenos Aires tuvo problemas sociales trascendentes que se presentaron como parte de la agenda del gobierno porteño.

[6] En 2018, los índices de pobreza (31,9\%) e indigencia (6,2\%) del Gran Buenos Aires (EPHINDEC, Partidos del GBA, $1^{\circ}$ semestre de 2018) casi triplicaban los que observábamos en la ciudad de Buenos Aires en esos años.

[7] Según los investigadores Zack, Schteingart y Favata (2020), que unificaron los criterios de medición para poder comparar las mediciones realizadas con metodologías diversas, en el segundo semestre de 2015 la tasa de pobreza argentina era de $26,9 \%$ mientras que la de indigencia era de 4,7\%. En el primer semestre de 2019 , ya era de $35,4 \%$ y 7,6\%, respectivamente.

[8] En la ciudad de Buenos Aires la cantidad de indigentes se duplicó entre 2015 y 2018 ( $\ll$ Se duplicó la indigencia en la Capital Federal en tres años y ya alcanza a 200 mil personas»,TN, 28/1/2019). En los partidos del GBA, sólo entre diciembre de 2018 y el primer semestre de 2019 el número de indigentes sumó más de 85 mil personas («En el conurbano, la pobreza llegó al $40 \%$ y la indigencia se acerca al 10\%», Portal información, 1/10/19).

[9] En 2016 el presupuesto del Ministerio de Desarrollo Social representaba un 5,78\% del total, cifra que se redujo en 2017 y que luego se aumentó al 6\% en 2018.

[10] Si comparamos este dato con el gasto en 2016 de las carteras de Salud, de Desarrollo Social y de la seguridad social, representaron en conjunto el $48,89 \%$ del total. Dicha cifra es similar porcentualmente a la unificada que aparece en el presupuesto de 2019, aunque en ese año pasó a depender toda del Ministerio de Salud y Desarrollo Social, mostrando cómo se robusteció el lugar de la ministra en el gabinete nacional en el último año del gobierno de Cambiemos.

[11] Más del 35\% de los funcionarios de CABA participó en alguna fundación en algún momento de su vida mientras que el conjunto más grande de individuos con experiencia previa de participación partidaria venía de las filas del peronismo (17,5\%).

[12] Mientras que un $19 \%$ de los funcionarios trabajaban inmediatamente antes en el sector privado, destacándose quienes ocupaban altos cargos empresariales, otro $19 \%$ estaba representado por individuos que fundaron o dirigieron ONGs ligadas a ese mundo empresarial. A diferencia de los cuadros sociales de la derecha de los años 60 (Giorgi, 2015; Osuna, 2016), las organizaciones católicas ocuparon un rol secundario en la sociabilidad profesional de buena parte de los funcionarios macristas.

[13] Mario Quintana es amigo personal de Horacio Rodríguez Larreta, a quien conoció en la Fundación Sophia y quien fomentó su ingreso a Cambiemos y al Poder Ejecutivo Nacional, donde ocupó el cargo de secretario de Coordinación Interministerial entre 2015 y 2018. Además, Quintana participa en Ashoka, organización de la que formó parte Matías Kelly, secretario de Economía Social del MDSN entre 2016 y 2019. Desde el año 2000 la trayectoria laboral de Quintana se vincula al Grupo Pegasus, del cual es socio y accionista, y que posee o poseía empresas como Freddo, Farmacity, Musimundo y empresas de alimentos, inmobiliarias, tecnología y otras, en Argentina y Colombia.

[14] Sólo 8 funcionarios del gabinete de Vidal disputaron las intendencias de distintos distritos del conurbano y, entre ellos, la mitad ocupaban cargos en el Ministerio de Desarrollo Social siendo esta cartera la más importante del gabinete en términos de reclutamiento de candidatos.

[15] Tanto para quienes importaron un saber técnico de gestión en programas sociales como para quienes valorizaron un saber managerial la concepción que primó se sostenía sobre la idea de que la aplicación de técnicas de gestión y medición mejorarían por sí mismo el desempeño del 
Estado. Esta concepción también primó en otras derechas latinoamericanas como muestra, por ejemplo, Rodríguez (2019) al analizar el caso de Perú donde la tecnocracia liberal, que gobernó directamente a partir de la elección de Pedro Kuczynski en 2016, jerarquizó esta mirada que despolitiza el Estado, sin discutir la representación popular o la toma de decisiones en el marco de conflictos.

[16] Al igual que otras derechas del continente, como Evópoli en Chile (Alenda, 2020), esta fuerza política y sus cuadros de gestión presentaban sus prácticas y discursos como nuevas formas de hacer política. 